\title{
Modelling phase change in a novel turbo expander for application to heat pumps and refrigeration cycles
}

\author{
E. Geoffrey Engelbrecht ${ }^{1, *}$, Zoitis Giakoumis ${ }^{1}$, Stathis Sidiropoulos ${ }^{1}$, Alexandros \\ Chasoglou ${ }^{1}$ and Ndaona Chokani ${ }^{1}$ \\ ${ }^{1}$ Limmat Scientific AG, Industriestrasse 7, CH-6300 Zug, Switzerland
}

\begin{abstract}
A novel turbo expander based on the Tesla turbine is proposed to be applied to a heat pump or refrigeration cycle to improve the overall cycle efficiency. Initial numerical modelling of this turbo expander at representative conditions was carried out using the homogeneous relaxation model (HRM) to assess the influence of phase change on performance. The presence of a dense cloud of liquid droplets within the rotor was predicted to produce a significant back pressure on the turbine nozzle postponing the phase change. This was expected to occur in the vicinity at the outlet of the nozzle, but high volume fractions of liquid was predicted to penetrate deeper inside the rotor, especially at higher RPM. The resulting lower velocities of the liquid flow at the inlet of the rotor was predicted to significantly degrades the performance of the turbine. It is thus important for a successful implementation of this concept to remove as much liquid droplets as possible before the flow enters the rotor in order to minimise the back pressure.
\end{abstract}

\section{Introduction}

In most commercial applications of a heat pump or refrigeration cycle an expansion valve (Fig. 1) is used between the condenser and evaporator. Thereby, work produced by the expansion of this flow, which might be partially captured by a turboexpander, is lost. One reason that turboexpanders are not commonly used in these cycles is that usually a partial phase change occurs during the expansion of the flow; the flow typically enters the nozzle primarily as liquid, by volume, and leaves primarily as vapour, by volume, containing a dense cloud of liquid droplets. In traditional turbomachinery liquid droplets are highly undesirable, as they cause erosion damage to turbine blades.

Based on the Tesla turbine, Spezia et al [1] proposed implementing a turboexpander in place of the expansion valve [2] (Fig. 2). This turbine consists of a series of closely spaced disks. Fluid tangentially enters the space between the disks through nozzles that are at the outer edge of the disks and exits from the space near the axis of the disks. Because of the tangential orientation of the nozzles, a vortical flow is generated between the disks. The disks pick up energy from the flow through the boundary layer and start spinning. Because

\footnotetext{
* Corresponding author: engelbrecht@limmatscientific.ch
} 
this turbine extracts energy from the boundary layer, the only surface that is potentially exposed to collision, and hence to erosion from liquid droplets, would be the outer edge of the disks.

Given that the local speed of sound depends strongly on the vapour fraction, and that the phase change has an impact on temperature and a strong impact on density, it is critical to accurately predict the rate of phase change within the expander in order to obtain reasonable predictions of turbine performance.

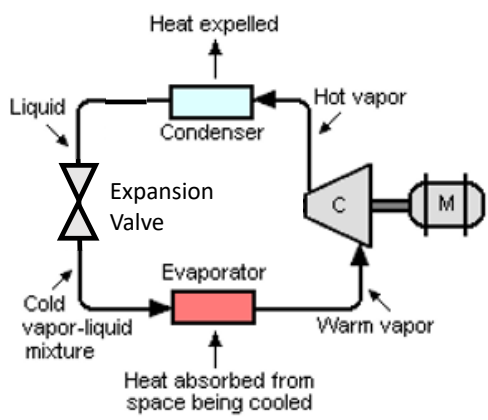

Common Commercial Refrigeration Cycle

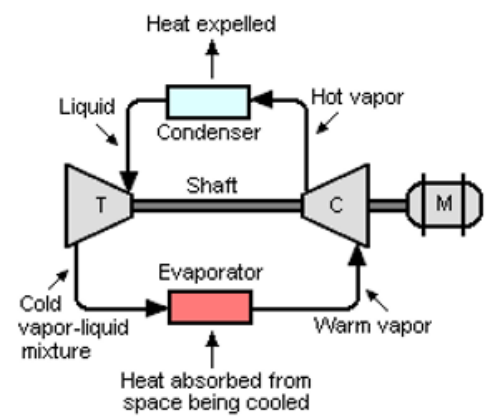

Refrigeration Cycle with a Turboexpander

$\mathrm{T}=$ Expansion turbine, $\mathrm{C}=$ compressor, $\mathrm{M}=$ Motor

Fig. 1. Refrigeration Cycles (proposed turbo expander, pictured on right, to provide mechanical power to supplement power requirement of compressor)
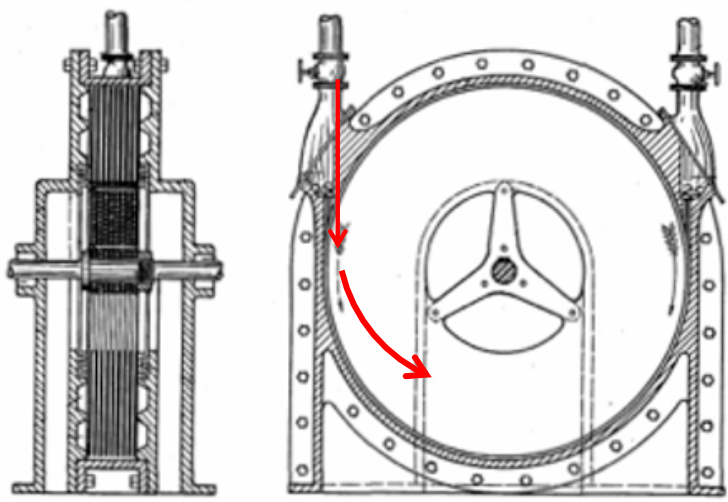

Fig. 2. Tesla Turbine

A Tesla turbine test rig comprised of a single passage between two disks and using steam as a working fluid has been developed to test this concept. Prior to the testing some initial modelling, which is reported here, was carried out to assess the expected performance of the test rig.

\section{Tesla Turbine Test Rig}

The test rig being analysed in this study is based on a static test rig being constructed at the University of Genoa shown in Fig. 3. This rig is designed to have maximum optical 
access, and thus has no rotating disks. However as the ultimate aim of the study is to design a working Tesla turbine, the modelling was also carried out for the case with rotating disks.

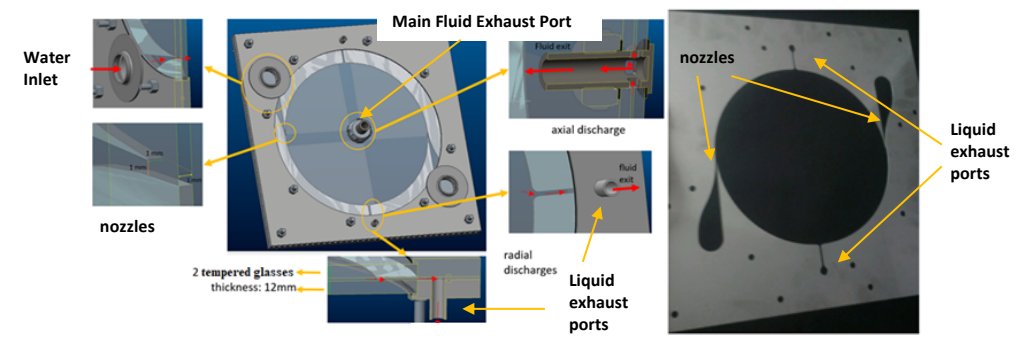

Fig. 3. Layout of Tesla turbine test rig

In the test rig liquid water at $0.4 \mathrm{MPa}$ and $140^{\circ} \mathrm{C}$ is supplied to two nozzles that are spaced $180^{\circ}$ apart. The majority of the resulting liquid vapour mixture exits the test rig at atmospheric conditions through a shaft in the centre of the rig. There are also two exhaust ports at the outer radius $90^{\circ}$ from each nozzle to remove liquid. The gap between the disks in the rig is $1 \mathrm{~mm}$ and the disks have a diameter of $100 \mathrm{~mm}$.

The idealised operating line of the lossless turbine can be seen in Fig. 4. It is expected that the steam exiting the turbine will have a quality of approximately $5 \%$.

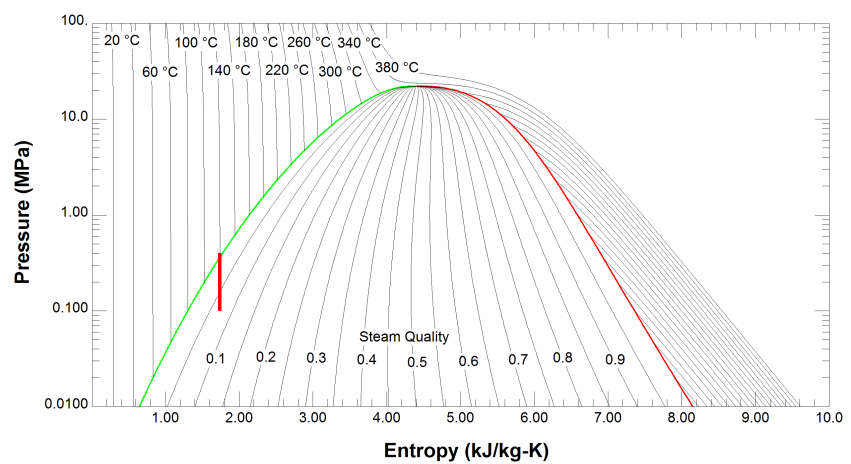

Fig. 4. Vertical red line shows expected operating line of the turbine with no losses

\section{Model}

CFX is used to solve the Reynolds averaged Navier Stokes equations. The SST (Shear Stress Transport) model [3] is used to close the Reynolds Stresses and the homogeneous relaxation model (HRM) is used to predict the rate of phase change. Water properties are modelled using the IAPWS IF97 steam tables.

The HRM has been applied successfully over the last decade to model flash atomisation in gasoline direct injection engines [4-12]. The HRM was first proposed by Bilicki et al [13] and later extended by Downar-Zapolski et al [14] to model the influence of non-equilibrium effects on the vapour formation rate during the flash boiling of water.

The model proposes that the deviation from equilibrium can be solved with an assumed linear approximation for the rate of change of the vapour mass fraction $y_{v}$ :

$$
\frac{D y_{v}}{D t}=\frac{\bar{y}_{v}-y_{v}}{\Theta}
$$


where $\bar{y}_{v}$ is the equilibrium vapour mass fraction and $\Theta$ is the relaxation time scale over which $y_{v}$ tends to $\bar{y}_{v}$.

The relaxation time $\Theta$ is modelled by an empirically derived correlation proposed by Downar-Zapolski et al [14] based on Reocreux's measurements of water flashing in a pipe for pressure drops below 10 bar [15].

$$
\Theta=\Theta_{0} \propto^{-0.257} \psi^{-2.24}
$$

where $\Theta_{0}$ is a constant $6.51 \times 10^{-4} \mathrm{~s}, \propto$ is the vapour volume or void fraction given by:

$$
\propto=\frac{y_{v} \rho_{l}}{\rho_{v}+y_{v}\left(\rho_{l}-\rho_{v}\right)}
$$

where $\rho_{l}$ and $\rho_{v}$ are the densities of the liquid and vapour phases. $\psi$ is the dimensionless pressure given by:

$$
\psi=\left|\frac{p_{s a t}-p}{p_{\text {sat }}}\right|
$$

where $p$ and $p_{\text {sat }}$ are the local static pressure and saturation pressure respectively.

\section{Analysis}

To validate the modelling approach, predictions were compared against measurements made by Park et al [16] at similar operating conditions for a simple rectangular nozzle which exhausted into a large chamber at atmospheric pressure. It was found in this work that the standard HRM predicted phase changes that were too far downstream. This mismatch may be in part due to the roughened surface of nozzle walls in the experiment, which are thought to have increased the number of nucleation sites for vapour bubble formation. A $\Theta_{0}$ which was 10 times smaller than that proposed for the standard HRM was found to produce the closest agreement to the measurements. Thus this value (HRM/10) was used for the analysis of the Tesla test turbine. Given the uncertainty of this model constant, sensitivity analysis of this parameter was performed by also carrying out calculations with values of $\Theta_{0} / 5(\mathrm{HRM} / 5)$ and $\Theta_{0} / 20(\mathrm{HRM} / 20)$.

Simulations were performed on a $180^{\circ}$ periodic sector of the proposed Tesla test turbine. A hybrid unstructured grid of 17 million nodes producing a y+ of less than 2 was used. A grid sensitivity study was not performed as the study was intended to be qualitative in nature however extensive experience from previous turbomachinery simulations was applied and the mesh resolution was adapted to resolve areas of the flow where steep gradients were expected. The liquid exhaust ports at the outside edge of the rotor were not considered in this initial study. Calculations with dry steam (assuming the same boundary conditions in terms of pressure and temperature but with water simulated as a vapour without phase change) as a working fluid provided a baseline. For simulations with phase change, only a single momentum equation was solved for both liquid and vapour phases. The influence of that assumption was investigated and is discussed later.

Fig. 5 shows that the predicted torque drops rapidly with phase change reaching zero at around 1500 RPM. There is very little influence of $\Theta_{0}$ for the values investigated.

The reason for the rapid drop in torque is shown in Fig. 6. As RPM increases the point at which the phase change starts moves downstream into the gap between the disks. Given the large difference in the densities of the liquid and vapour, at high RPM this results in 
negative relative velocities in a region that is near the tip of the disks; this region acts as a brake on the disk.
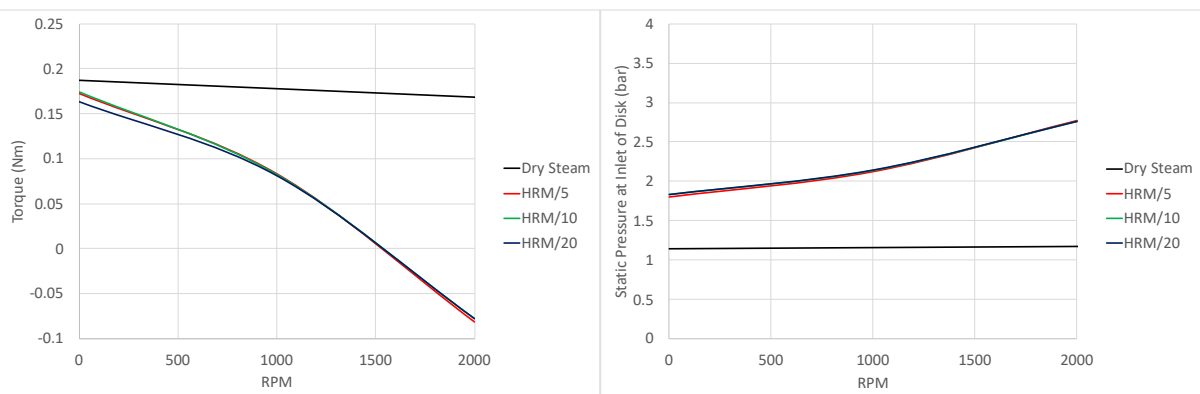

Fig. 5. Left: predicted torque versus RPM, Right: static pressure at inlet of disk versus RPM
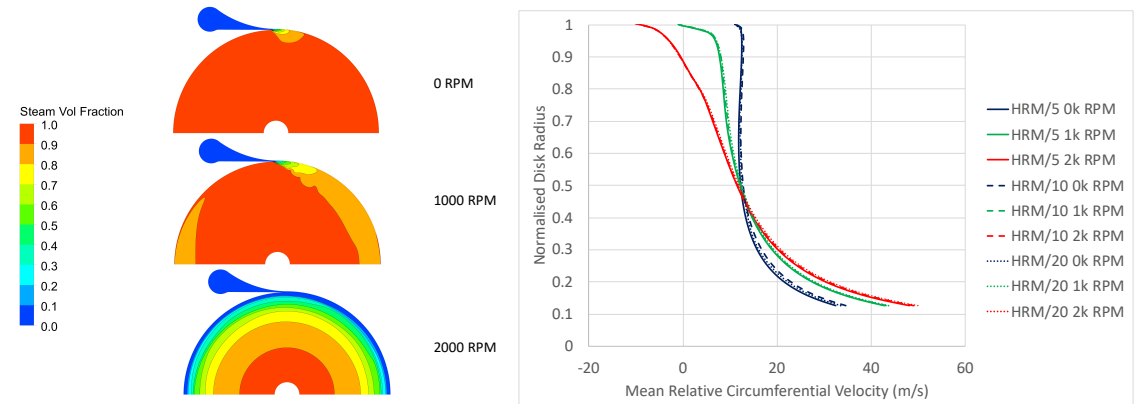

Fig. 6. Left: predicted vapour volume fraction as a function of RPM (HRM/10), Right: circumferentially averaged relative circumferential velocity (i.e. circumferential velocity minus the disk velocity) versus disk radius

The primary reason the phase change is pushed downstream relates to the influence of the centrifugal force, produced by the vortical flow between the disks, on the back pressure of the flow exiting the nozzle. Given that the average density of the wet steam is substantially higher than the density of dry steam this is significant when liquid droplets are present in the flow (Fig. 5).

Because of time and computational constraints it was not possible to carry out a series of volume of fluids simulations to predict the droplet sizes and a satisfactory approach to do this reliably in a less computationally expensive way is still to be found. Therefore to investigate the influence of inhomogeneity in the momentum between the two phases a separate investigation was carried out for a periodic $5^{\circ}$ sector of the disk.

These simulations were performed on a grid containing 600'000 nodes producing a y+ of less than 2 and were carried out without phase change and with a uniform inlet mass flow and vector direction applied at the inlet to the gap between the disks which was extracted from the $180^{\circ}$ sector simulations. The steam quality was assumed to be $5 \%$ at the inlet. The liquid droplets were assumed to have a uniform size and the sensitivity of the results to this size was explored.

For small droplets $(\leq 12.5 \mu \mathrm{m})$ and low RPM, an Eulerian-Eulerian approach was used to model the liquid and vapour phases, whereas for larger droplets and higher RPMs, a Lagrangian-Eulerian approach was adopted. The primary reason for using these two different approaches is that the Lagrangian-Eulerian approach in CFX does not account for the volume displacement of the liquid phase which becomes important as the liquid volume fraction increases. The Eulerian-Eulerian approach which does account for this volume displacement 
has more difficulties when there is a significant separation in the momentum between the two phases, which is particularly true when the liquid droplets reverse direction and are thrown radially outwards.

The centrifugal forces have a stronger effect on the denser liquid phase than the less dense vapour phase causing the liquid droplets to take longer to reach the outlet at the centre of the disk. This tends to concentrate more liquid, thereby reducing the vapour volume fraction, and increasing the average density of the flow. The result of this is a higher back pressure at the outlet of the nozzle (Fig. 7). The larger is the droplet diameter and the higher is the RPM then the stronger is this effect. Above a certain droplet size and RPM, the droplets eventually centrifuge to the outer radius exiting the domain and do not penetrate significantly into the gap between the disks. At 0 RPM this is predicted to already occur for droplets exceeding $40 \mu \mathrm{m}$, but will occur for smaller droplets at higher RPMs with the point at which it occurs being inversely related to the droplet size. It is not easy to precisely predict the point in terms of droplet diameter and RPM when this transition occurs as both modelling approaches become unstable near this transition.

Eliminating the liquid phase altogether before the flow enters the disks (shown in Fig. 7 as vapour only) significantly reduces this back pressure. Thus, provided that the droplets formed are large enough or that the RPM is high enough, it should be possible to remove the majority of the liquid at the outer radius of the turbine through the liquid extraction ports and reduce the negative impact of the droplets on the pressure drop across the rotor. If the droplets formed are too small it may be necessary to implement an alternative method of separating the droplets from the flow prior to the flow's entry into the gap between the disks.
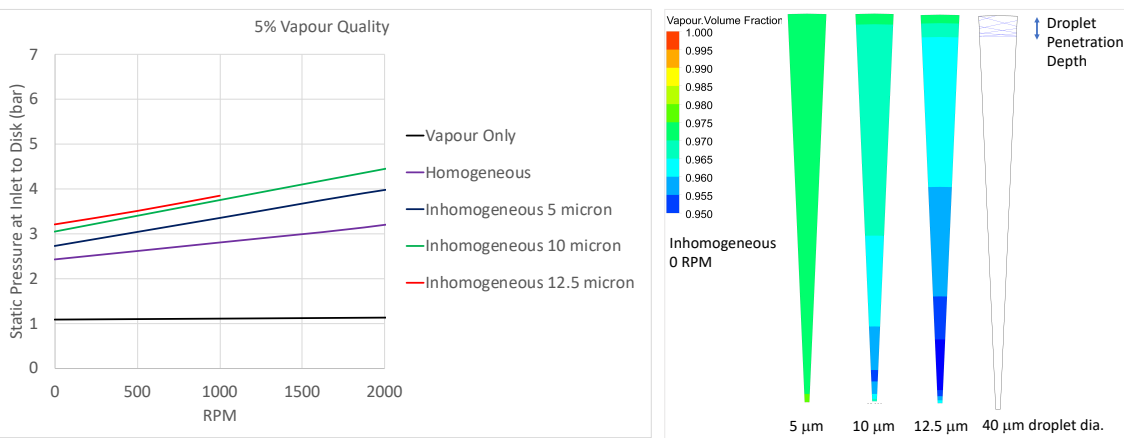

Fig. 7. Left: influence of the presence of liquid and inhomogeneity on disk inlet pressure versus RPM, Right: influence of droplet size on liquid distribution

\section{Conclusions}

A novel application of a Tesla turbine to the expansion of a heat pump or refrigeration cycle is explored numerically in preparation for development testing of such a concept.

The presence of a dense cloud of liquid droplets in the flow between the disks is expected to produce a significant pressure drop across the rotor, which postpones the phase change and significantly reduces the performance of the turbine. If the droplets in this cloud are large enough or if the RPM of the disk is high enough, these droplets are expected to centrifuge to the outside of the disk and can potentially be removed through liquid extraction ports placed there. However if the liquid droplets are small, other means of liquid extraction may be necessary before the flow is admitted into the gap between the disks in order to avoid the large back pressure which would likely be produced by this flow.

Testing is expected to start shortly and this should confirm the size of the droplets as well as the findings of the initial modelling. The next steps in the modelling will be to investigate 
the efficacy of the liquid extraction ports which should help reduce the back pressure produced by the vapour liquid flow in the rotor and improve the performance of the turbine.

This project has received funding from the European Union's Horizon 2020 research and innovation programme under grant agreement No 764706, PUMP-HEAT project

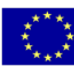
(https://www.pumpheat.eu/).

\section{References}

1. R.A. Spezia, A. Traverso, S. Barberis, L., Larosa, P. Silvestri, Patent WO 2018/127445 A1 (2018)

2. N. Tesla, Patent US 1061206 (1913)

3. F.R. Menter, AIAA Journal, 32, 1598 (1994)

4. S. Negro, G.M. Bianchi, Int. J. Therm. Sci., 50, 1460 (2011)

5. M. Moulai, R. Grover, S. Parrish, D. Schmidt, SAE Technical Paper 2015-01-0944 (2015)

6. E.T. Baldwin, R.O. Grover, S.E. Parrish, D.J. Duke, K.E. Matusik, C.F. Powell, A.L. Kastengren, D.P. Schmidt, Int. J. Multiph. Flow, 87, 90 (2016)

7. P. Strek, D. Duke, A. Swantek, A. Kastengren, C.F. Powell, D.P. Schmidt, SAE Technical Paper 2016-01-0858 (2016)

8. K. Saha, S. Som, M. Battistoni, Y. Li, S. Quan, P.K. Senecal, Proceedings of the ASME 2015 Internal Combustion Engine Division Fall Technical Conference ICEF2015-1112 (2015)

9. K. Saha, S. Som, M. Battistoni, Y.Li, E. Pomraning, P.K. Senecal, SAE Technical Paper 2016-01-0870 (2016)

10. K. Saha, S. Som, M. Battistoni, Atomization Spray., 27, 345 (2017)

11. K. Saha, S. Quan, M. Battistoni, P. Senecal, SAE Technical Paper 2017-01-0834 (2017)

12. S.K. Rachakonda, Y. Wang, D.P. Schmidt, Atomization Spray., 28, 111 (2018)

13. Z. Bilicki, J. Kestin, Proc. Royal Soc. A, 428, 379 (1990)

14. P. Downar-Zapolski, Z. Bilicki, L.Bolle, J. Franco, Int. J. Multiph. Flow, 22, 473 (1996)

15. M. Reocreux, Contribution a l'etude des debits critiques en ecoulement diphasique eauvapeur, PhD Thesis, Université Scientifique et Medicale de Grenoble (1974)

16. B.S. Park, S.Y. Lee, Atomization Spray., 4, 159 (1994)

17. Y. Liao, D. Lucas, Int. J. Heat Mass Transf., 111, 246 (2017) 